\title{
A research survey on scheduling techniques in lte-based network and its state of art
}

\author{
Srinivasa R K*1, Hemantha Kumar A.R ${ }^{2}$ \\ ${ }^{1}$ Visvesvaraya Technological University, Department of Electronics \& Communication Engineering, \\ BIT, Bangalore, India \\ ${ }^{2}$ Department of Electronics \& Communication Engineering, \\ BIT, Bangalore, India \\ *Corresponding author, e-mail: srinirkwork@gmail.com
}

\begin{abstract}
The growing interest over the mobile based communication has attracted the Long-Term Evolution (LTE) technique which facilitates higher data transmission rates to its dynamic users. The LTE-based network involves with scheduling which governs the better communication at different traffic (large, small, medium) condition. The LTE caters up network demands and encounters some potential and unreported problems in presence of large traffic with uncertainty of its associated load. Thus, scheduling becomes most essential is must. This manuscript reviews the recent existing researches with their contribution along with significances and limitations. The paper also contributes in identify the state of art in research issues to identify the research gap towards scheduling techniques in LTE.
\end{abstract}

Keywords: 4G, Downlink Transmission, Long-Term Evolution (LTE), Scheduling, Uplink Transmission

Copyright $(2) 2018$ APTIKOM - All rights reserved.

\section{Introduction}

The Long-Term Evolution (LTE) is aims to cater up the exponentially increasing demands of the dynamic users of cellular network [1]. LTE offers maximized rate of transmission with much reduced network delay and simplified operational cost [2]. The LTE scheduling process in LTE is used to get proper resource allocation to assist the variable demands of resources during data transmission process [3]. The scheduling process is designed for considering the explicit condition of the communication channel, volume of traffic, and quality of service [4]. A good scheduler will have a potential dependencies on gain factor involved in channel state associated with each users as well as sub-carriers. To offer lower delay in scheduling process, the standards of LTE claims of offering $1 \mathrm{msecs}$ of duration involved in data forwarding process [5]. The scheduling processes are always considered to be carried out over base station. While executing the scheduling decision, the base station performs allocation of resources considering both downlink as well as uplink transmission [6-7]. Currently, LTE uses Orthogonal Frequency Division Multiplexing (OFDM) or OFDM Access (OFDMA) for uplink transmission while the downlink transmission is assisted by Single-Carrier Frequency Division Multiple Access (SC-FDMA). The scheduling in LTE is very much different from other form of technologies of accessing, where LTE based scheduling are carried out considering both time and frequency domain for users while other access technologies are limited to only time domain [8]. The problem associated with the scheduling process in LTE is all about identifying the precise allocation of scheduling blocks to specific user equipment's for the purpose of increasing cumulative throughput or minimizing network delay [9]. In order to avoid any issues, the present research work towards schedulers are designed to be functional in two process i.e. scheduling packet using time domain first and then execute frequency domain [10-20]. There also existing certain research work that uses multiple-input multiple-output as a scheme to design scheduler for LTE networks [21-22]. There has been various work carried out towards LTE network; however; various problems and issues are still demanding more investigation. The existing research work are not found to report any form of extensive supportability of spatial multiplexing during both uplink and downlink transmission for more number of layers in order to increase the transmission rates. There is also a significant problems associated with both the uplink and downlink transmission when they were made to work in multipoint forwarding and receiving of packets for increasing the throughput and coverage of extended rate of data. It is also found that adoption of relaying methodologies towards improving the optimal usage of communication channel 
as well as its associated capacity has been less explored. All these problems are unsolved and unaddressed and require a good series of dedicated investigation in order to explore a better form of solution.

Hence, this paper reviews the existing approaches towards scheduling process in LTE-based network and deliberately attempts to identify the potential research gap. The idea is to assist the upcoming researchers to measure the level of effectiveness in existing scheduling techniques. The organization of this paper is as follows: Section 2 discusses about the theoretical briefing of LTE followed by exhaustive review of existing scheduling practices in Section 3. Section 4 discusses about research gap while Section 5 briefs about the contribution of this paper followed by tentative future work direction. The next section discusses about the existing approaches used to address the scheduling problems in LTE.

\section{RESEARCH TOWARDS SCHEDULING PROBLEMS}

This section discusses about the existing research-based approaches towards enhancing the scheduling process in LTE-based Networks. Aiyetoro et al. [23] have introduced a scheduling plan in satellite of LTE networks to achieve the ideal QoS of LTE standards in respect of throughput and delay constraints. Here, a non-linear optimization approach is used and compared their presented approach with other optimal scheduling method. The [23] system achieves good throughput and gives optimal scheduling performance. Chen and Liu [24] gives a scheduling model for media streaming transmission to achieve play-out continuity over LTE services. The [24] model is based on discontinuous reception called as DRX and playout buffer with including three methods to solve this problem that are 1). Remaining playout time, 2). Resource allocation technique and 3). Buffer level calculation technique. The outcome reduces the energy cost of user equipments (UEs) at low level and it is also shown that performance of presented model is $50 \%$ better when compared with existing scheme. Fan et al. [25] works on multiuser scheduling and pairing system with including inter and intra cell interference in the LTE uplink

cellular networks. Here, a collaborative optimization problem is defined by considering signal-tointerference-plus-noise ratio (SINR) into SC-FDMA and frequency domain minimum mean- square error (FD-MMSE) in concept of extending throughput of overall network. The study outcomes display that the presented method reduced the interference and perform well in compared to existing method. Ferdosian et al. [26] considered difficulty in scheduling for heterogeneous traffic in overload phase in LTE download link in which user's channel details are varying and is not fixed. Here, an analytical framework is considered and presented a Fractional Knapsack algorithm to achieve QoS requirement and for efficient consumption of resource for heterogeneous traffic in overload state of cell. The results gives suitable fairness and fullfill QoS requirements.

Ghavimi et al. [27] considered issue related with the power allocation, Resource block (RB) and QoS requirement for M2M communication in uplink SC-FDMA dependent LTE-A networks. The authors formulate computational algorithm based on Lagrange multiplier concept. The outcomes gives that the [27] method defeats the existing Greedy algorithm in respect of providing maximum throughput and meets the QoS requirements. Grondalen et al. [28] introduced a scheduler performance analysis parameter of different-different LTE downlinks channels with flat and frequency selective channels, saturated UDP and TCP traffic source and also considering the frequency-domain and time-domain schedulers. The outcomes better tradeoff among the cell capacity and fairness for UDP and TCP traffic source. Guo et al. [29] considered multi user multiple-input multiple outputs (MIMO) scheduling in LTE downlink cellular networks. The framework is developed in multi user MIMO system in presence of inter stream interference and inter user interference and developed a optimization problem approach based on precoding matrix indicator selection, joint user pairing and RB allocation and considering these parameter optimal scheduling algorithm is elaborated which used to increase throughput of overall system. The simulated results display that it is able to achieve good throughput.

Ko et al. [30] has designed scheme in which channel selection and frame scheduling taken as joint optimization problem approach which bring maximum throughput in LTE and also balancing the fairness among the WLAN and LTE. The authors have formulate another heuristic algorithm named as sequential operational algorithm (SOA) to reduce the complexity of The authors discovered approach and in SOA the channels are selected according to channel ideal state probability and LTE transmission interval can be calculated by fairness between the LTE and WLAN. The outcomes of this study are display that the presented method gets comparable throughput to optimal solution and also get fairness between LTE and WLAN. Li et al. [31] has introduced with overview on MIMO techniques and multi-cell coordinate scheduling technique for LTE cellular networks. The authors have considered various approaches on SU-MIMO and multi-user MIMO for improving more performance parameter and discussed minimization of inter-cell interference methods for uplink and downlink in LTE. The study reveals that many aspects are

APTIKOM J. CSIT Vol. 3, No. 2, 2018: $51-58$ 
discussed in terms of improving performance parameter.Lien et al. [32] has concentrated on Licensed-assisted access (LLA) collocated with wireless networks in which the authors developed optimum radio access which display the dynamic switch between scheduling dependent radio access and a random access plans. The result of this study shows the most favorable design of LLA networks.

Liu and Chen [33] have developed a framework based on downlink scheduling for enhancing and handle several complexities of media communication transmission using LTE network services by considering the following control module such as transmission control module and smart resource allocation module. The study outcomes show that discussed approach gives suitable scheduling performance for multimedia transmission over LTE networks.Luo et al. [34] has offered a new scheme which involved component carrier on/off scheduling and resource allocation. The presented scheme is based on the Lyapunov optimization to enhance the activity of packet throughput and uses Vapnik-Chervonenkis theoretical conept to determine the variation between predicted optimal value and practical value. The simulate outcomes of study generates good trade-off among the power utilization and packet throughput at the time of low traffic load. Mehaseb et al. [35] has studied on various uplink scheduling methods in LTE in regards of M2M communication for providing QoS requirements, power efficiency, scalability and multi-hop connectivity. The outcome this study is shows that author have tried to flash some light on the construction and designing requirement of scheduling techniques for M2M communication to meet the goal of QoS, throughput and delay constraint. Minelli et al. [36] has reported the performance parameter of two conventional scheduler methods that are Proportional Fair (PF) and Round Robin (RR) for relay-enhanced LTE-A cellular networks. The authors have formulate the optimal model for extending the throughput and designed sub-optimal relay nodes placement for comparing RR and PF. The outcomes of study shows that PF is not performed well with respect of RR in several scheme.

Mostafa and Gadallah [37] have presented scheduling methodology to analyze the important factor of information in the data to be sent by machine type communications devices. (MTCDs). A statistical priority approach is used as the scheduling algorithm to define the scarce radio resources to MTCDs. The results offer that the presented method achieves great performance then other baseline schedulers in such a way that it having the least number of deadline misses for critical data packets. Pan et al. [38], has carried out to work on improve the network traffic and enhance the network capability for LTEadvanced (LTE-A) networks. A novel Downlink Traffic Arrangement approach is introduced to adopt arrangement downlink traffic with double connectivity improvement for LTE-A small network. The simulation outcomes show that novel scheduling approach can successfully improve the throughput of network with double connectivity enhancement for LTE-A small network. The paper has presented by Ragaleux et al. [39] discuss about the frequency and time resulting issues in LTE-A network. To resolve these issues, introduced an approach is Robust and Probable Conscious Scheduling, which protect an extensive range of scheduling objectives. The proposed method developed by three algorithms is follows:

For problem reduction.

For First distribution and Users selection.

For potential-conscious allocation.

The suggested scheduling approach is more effective than traditional approaches in terms of satisfied operators in performance calculation outcomes. In the same way, Opportunistic Packet Delay Outage Ratio Aware function exceed the Proportional fair/MaxSNR effectiveness function for multimedia scenario. Wang and Tsai [40], has discussed about less system performance and lesser operator revenue problems arises in LTE network. In this study, authors has addressed a framework model is Pricing-conscious Resource Scheduling to resolve this conflict. It contain pricing and arrangement both module. Without loss of generalization, the framework model categorize into three levels:

- Golden (high)

- $\quad$ Silver (medium)

- $\quad$ Bronze (low)

The result shows that the framework model make an optimal balance between revenue and performance. It also supports Quality of Services for video service and Voice over Internet Protocol. Zolfaghari and Taheri [41], has addressed a non-real time flows issues. To tackle this issue here, authors introduced a novel scheduling approach is Queue-conscious Channel-adapted method in performance enhancement. It is well-matched with congestion control algorithms like minimal cost flow control algorithm, which considered on fluid flow statement. By the using proposed approach provide higher performance as compared to plain scheduling methods. The outcomes shows the proposed scheduling technique with minimal cost flow control achieved high throughput and improve the elastic traffic. 
The study of Zolfaghari and Taheri [42], addressed issues in joint arrangement and congestion control optimization in LTE network. To improve these issues in LTE network explained about the scheduling methods which is Price Channel Quality. The outcomes displays an optimal adjustment between the entire throughput and the equality for the joint algorithm, where scheduling and congestion control distinctly designed and executed without any support. In the study of Masegosa and Gzalvez [43], has introduced the first appraisal of the operation and performance of LTE vehicle-to-vehicle mode 4 communication in accurate traffic conditions. In this study, author has introduced a novel technique is semi persistent scheduling based on sensing. It analyzed and measured the different types of transmission errors. The result shows the errors below $5 \%$ for all outcomes. The proposed technique provide reduction with the distance between receiver and transmitter and the channel load. In the study of Chang et al. [44] discussed the issues low throughput and high packet loss rate, which arises in LTE-A. Has introduced a technique which is based on cross layer packet scheduling, and it is classified into two mechanisms:

- $\quad$ Adaptive reward priority scheduling in MAC layer.

- $\quad$ Dynamic resource scheduling algorithm in physical layer.

The technique provides the collaboration with the data symbol scheduling and Channel Quality Indicator at physical layer. The outcomes shows given technique provide optimal solution for higher throughput and low packet delay in LTE-A network system. Kim and Park [45] has carried out the work on developed a framework an integrated modelling based on two dimensional Markov chain model for probability distribution of the number of arranged resource blocks. The outcomes defined that the presented framework model are reliable with spontaneous speculations. But it is tough to evaluate or examine the number of arranged resource blocks exactly. The study of Chakrapani [46] has described an efficient resource scheduling framework model, which is collaborate with narrowband internet things and improved machine type communication and also using two algorithmic approaches. The proposed technique reduces the complexity of limited bandwidth and improve the coverage LTE network. The outcomes shows that algorithmic approaches provides important profits increase to $80 \%$ over a loop based scheduling techniques and also the presented algorithmic approach are generic and appropriate to enhanced narrowband and user equipment implementation. The study carried out of Feng et al. [47] has addressed the scheduling algorithms and Quality of Services based LTE downlink scheduler for improving low communication and application information in smart grid communication. The simulation outcomes display the improvement of packet loss rate, delay and average throughput. It is important enhancement in smart grid which based on LTE network. Alaa et al. [48] carried out the work on machine to machine delay sensitive and lack of standardization problems in LTE network system. So authors has presented a technique is dynamic access grant time interval control scheduling approach which is based on non-preemptive queuing framework. The introduced scheduling approach has achieves an optimal percentage in reducing delay and as well as packet loss decreases in less percentage. Lau et al. [49], has introduced a framework model for inefficient radio resource usage in LTE broadcasting. The framework which defined by author is Audience Driven Live Television Scheduling method, to enhance radio resource usage for television broadcasting over LTE network system. The proposed framework method usage three algorithms:

For television channels popularity calculation.

For resource approximation.

For resource allocation.

The framework method is structured for both rural and suburban areas and it is easy to relocate in different locations. The outcomes shows that the proposed method attains higher broadcast proficiency and conserved important amount of radio resources. Cano et al. [50], has described the issue of low throughput and high delay cost of heterogeneity. The method is introduced by authors are Listen before Talk with Load Based Equipment (LT/LBE) and Carrier Sensing and Adaptive Transmission (CSAT) approach. The experimental outcomes shows the heterogeneity cost is more for CSAT, and throughput of LTE is less when compared to use of LT/LBE. In defective inter technology detection the consumption of explicit communication is more difficult in CSAT due to higher possibility of loss compared to LT/LBE. Eladham et al. [51], has discussed about lower system performance in-forms of spectral proficiency and lower throughput issues. For this issues introduced an algorithmic design of Improved Capacity Superior Proportional Fair downlink scheduling methods for achieve optimal system performance. It helps to enhance the heterogeneous network performance that allocate resource blocks with extend the system capacity and high fairness. The outcomes of this study shows that proposed algorithm enhance spectral proficiency and fairness as compared to other traditional approaches. Similarly, Xitlaly et al. [52] have also addressed scheduling problems. Hence, all the above mentioned techniques offers solutions towards

APTIKOM J. CSIT Vol. 3, No. 2, 2018: $51-58$ 
scheduling-problems. Table 1 summarizes the effectiveness of existing approaches towards scheduling in LTE-based networks.

Table 1. Summary of Existing Approaches

\begin{tabular}{|c|c|c|c|}
\hline Authors & Methods & Advantages & Limitations \\
\hline $\begin{array}{l}\text { Aiyetoro et } \\
\text { al.[23] }\end{array}$ & $\begin{array}{l}\text { Non-linear optimization } \\
\text { approach }\end{array}$ & $\begin{array}{l}\text { Ideal throughput and supports both } \\
\text { single and multiple traffic state. }\end{array}$ & $\begin{array}{l}\text { Only achieves maximum } \\
\text { throughput. }\end{array}$ \\
\hline $\begin{array}{l}\text { Chen and Liu } \\
{[24]}\end{array}$ & $\begin{array}{l}\text { Playout buffer and DRX } \\
\text { based mechanism. }\end{array}$ & $\begin{array}{l}\text { Playout continuity in steaming and } \\
\text { saves UEs energy. }\end{array}$ & $\begin{array}{l}\text { Insufficient to keep Quality of } \\
\text { experience of client. Or limited } \\
\text { power saving. }\end{array}$ \\
\hline Fan et al. [25] & Optimization Algorithm & $\begin{array}{l}\text { Improved performance in LTE } \\
\text { uplink cellular networks. }\end{array}$ & No benchmarking \\
\hline $\begin{array}{l}\text { Ferdosian et } \\
\text { al.[26] }\end{array}$ & $\begin{array}{l}\text { Analytical framework and } \\
\text { Fractional Knapsack } \\
\text { algorithm. }\end{array}$ & $\begin{array}{l}\text { Maximum fairness and balanced } \\
\text { QoS including throughput and } \\
\text { delay. }\end{array}$ & $\begin{array}{l}\text { Less focus on practical } \\
\text { implementation }\end{array}$ \\
\hline $\begin{array}{l}\text { Ghavimi et al. } \\
\text { [27] }\end{array}$ & $\begin{array}{l}\text { Binary integer programming } \\
\text { and Lagrange concept. }\end{array}$ & $\begin{array}{l}\text { Maximum throughput and less } \\
\text { delay. }\end{array}$ & $\begin{array}{l}\text { Only applicable for uplink } \\
\text { transmission }\end{array}$ \\
\hline $\begin{array}{l}\text { Grondalen et al. } \\
{[28]}\end{array}$ & $\begin{array}{l}\text { Performance parameter } \\
\text { analysis approach }\end{array}$ & $\begin{array}{l}\text { Better tradeoff between cell } \\
\text { capacity and good fairness for TCP } \\
\text { and UDP. }\end{array}$ & Results not benchmarked. \\
\hline Guo et al. [29] & $\begin{array}{l}\text { Optimal scheduling } \\
\text { algorithm. }\end{array}$ & $\begin{array}{l}\text { Achieves better throughput for all } \\
\text { network system. }\end{array}$ & Complexity not studied \\
\hline Ko et al. [30] & $\begin{array}{l}\text { Sequential operation } \\
\text { algorithm }\end{array}$ & $\begin{array}{l}\text { Get good throughput and good } \\
\text { fairness between LTE and WLAN. }\end{array}$ & Results not benchmarked. \\
\hline $\begin{array}{l}\text { Lien and Liang } \\
\text { [31] }\end{array}$ & Optimum radio access & $\begin{array}{l}\text { Suggest optimum design for LLA } \\
\text { networks. }\end{array}$ & Less exhaustive investigation \\
\hline Liu et al. [32] & $\begin{array}{l}\text { Analytical modeling, hand- } \\
\text { off }\end{array}$ & Efficient resource utilization & Complexity not studied \\
\hline Luo et al.[33] & Learning-based & Energy efficient & Narrowed test-environment \\
\hline $\begin{array}{l}\text { Mehaseb et al. } \\
\text { [34] }\end{array}$ & Energy-efficient modeling & Support multi-hop & Results not benchmarked. \\
\hline Minelli et al.[35] & Statistical modeling & Supports multiple traffic system & $\begin{array}{l}\text { Results not benchmarked., includes } \\
\text { overhead }\end{array}$ \\
\hline $\begin{array}{l}\text { Mostafa et al. } \\
\text { [36] }\end{array}$ & Statistical modeling & Supports multiple traffic system & $\begin{array}{l}\text { Results not benchmarked., includes } \\
\text { overhead }\end{array}$ \\
\hline Pan et al.[37] & $\begin{array}{l}\text { Analytical modeling, } \\
\text { splitting approach }\end{array}$ & Enhanced network throughput & Restricted to small cell only \\
\hline Pan et al. [38] & $\begin{array}{l}\text { Downlink Traffic } \\
\text { Arrangement }\end{array}$ & $\begin{array}{l}\text { Increase throughput, double } \\
\text { connectivity }\end{array}$ & No benchmarking, high delay \\
\hline $\begin{array}{l}\text { Ragaleux et al. } \\
\text { [39] }\end{array}$ & $\begin{array}{l}\text { Robust and Probable } \\
\text { Conscious Scheduling }\end{array}$ & Low packet delay & $\begin{array}{l}\text { Doesn't emphasize on device } \\
\text { heterogeneity }\end{array}$ \\
\hline $\begin{array}{l}\text { Wang and Tsai } \\
{[40]}\end{array}$ & $\begin{array}{l}\text { Pricing-conscious Resource } \\
\text { Scheduling }\end{array}$ & $\begin{array}{l}\text { High throughput for voice and } \\
\text { video service over internet }\end{array}$ & No benchmarking \\
\hline $\begin{array}{l}\text { Zolfaghari and } \\
\text { Taheri [41] }\end{array}$ & $\begin{array}{l}\text { Queue-conscious Channel- } \\
\text { adapted method }\end{array}$ & High throughput, traffic control. & No benchmarking \\
\hline $\begin{array}{l}\text { Zolfaghari and } \\
\text { Taheri [42] }\end{array}$ & $\begin{array}{l}\text { Price Channel Quality } \\
\text { scheduling method }\end{array}$ & $\begin{array}{l}\text { Optimal throughput, designing and } \\
\text { implementation without any } \\
\text { support }\end{array}$ & $\begin{array}{l}\text { Outcomes not compared with any } \\
\text { existing system }\end{array}$ \\
\hline $\begin{array}{l}\text { Masegosa and } \\
\text { Gzalvez [43] }\end{array}$ & semi persistent scheduling & Simplified Technique & $\begin{array}{l}\text { Will lead to complexity and } \\
\text { overhead for large network }\end{array}$ \\
\hline Chang et al. [44] & $\begin{array}{l}\text { Adaptive reward priority and } \\
\text { Dynamic resource } \\
\text { scheduling }\end{array}$ & $\begin{array}{l}\text { Low packet delay, higher } \\
\text { throughput. }\end{array}$ & Lack of benchmarking \\
\hline $\begin{array}{l}\text { Kim and Park } \\
{[45]}\end{array}$ & $\begin{array}{l}\text { An integrated modelling } \\
\text { based on 2D Markov chain } \\
\text { model. }\end{array}$ & $\begin{array}{l}\text { Reliability with spontaneous } \\
\text { speculations. }\end{array}$ & Less effective on analyzing RBs. \\
\hline Chakrapani [46] & Algorithmic approach & $\begin{array}{l}\text { Profits increase to } 80 \% \text { over a loop } \\
\text { based scheduling. }\end{array}$ & Narrowed test environment \\
\hline Feng et al. [47] & $\begin{array}{l}\text { Quality of Services based } \\
\text { LTE downlink scheduler }\end{array}$ & $\begin{array}{l}\text { Average delay and Packet loss rate, } \\
\text { high throughput. }\end{array}$ & $\begin{array}{l}\text { Focuses only on downlink } \\
\text { transmission }\end{array}$ \\
\hline Alaa et al. [48] & $\begin{array}{l}\text { dynamic access grant time } \\
\text { interval control scheduling }\end{array}$ & $\begin{array}{l}\text { Reduce delay and low percentage } \\
\text { of packet loss. }\end{array}$ & $\begin{array}{l}\text { Performance depends on } \\
\text { hypothetical threshold }\end{array}$ \\
\hline Lau et al. [49] & $\begin{array}{l}\text { Audience Driven Live } \\
\text { Television Scheduling }\end{array}$ & $\begin{array}{l}\text { Easy to relocate different places, } \\
\text { higher broadcast efficiency. }\end{array}$ & Lack of benchmarking \\
\hline Cano et al. [50] & Analytical approach & Considers heterogeneity factor & Leads to complexity in longer run \\
\hline $\begin{array}{l}\text { Eladham et al. } \\
\text { [51] }\end{array}$ & Downlink scheduling & $\begin{array}{l}\text { Studies both homogeneous \& } \\
\text { heterogeneous network }\end{array}$ & Model validation missing \\
\hline $\begin{array}{l}\text { Xitlaly et al. } \\
\text { [52] }\end{array}$ & $\begin{array}{l}\text { Propagation model, round } \\
\text { robin }\end{array}$ & $\begin{array}{l}\text { Simplified approach, better } \\
\text { throughput }\end{array}$ & Narrowed test environment \\
\hline
\end{tabular}




\section{RESEARCH GAP}

On reviewing of existing researches in scheduling, following advantages and limitations. However, some of problems were exist during the implementation but they were not reported to be addressed. Following are the some research gaps identified after reviewing the existing approaches of scheduling in LTE-based networks.

- OFDM Problems Deemed Low Importance: A closer look into the existing system shows that consideration of OFDM has been less utilized after 3G-based network whereas in reality OFDM is highly essential for downlink transmission in LTE while the uplink transmission in LTE uses other mechanism (i.e. SC-FDMA). Adoption of OFDM in LTE degrades the scheduling decision in presence of large scale and dynamic traffic situation, which requires an immediate attention.

- Discrete Perspective of Delay: Irrespective of different number of research work addressing delay problem in LTE-based network, it has been observed that such research work has not considered the complexity in characterizing network delay as such network delay originated from the dynamic queues encounters potential challenge in design phase. It is because they cannot be realized using frequently utilized Markov chain. Such problem takes challenging shape when subjected to error-prone channels with multiple numbers of users in it.

- Least Complexity Analysis: The existing scheduling approaches towards LTE-based network have analyzed its effectiveness only on the basis of performance parameters e.g. throughput, CDF, delay, energy, etc. However, there is no such evidence if such techniques can be practically executed in realtime scenario as it lacks any form of complexity analysis.

- Lower Emphasis to Queue Management: A queue always exists between server and users, which are less likely to be seen to be discussed in existing literatures. Existing default queue management system only considers delay associated with one targeted server ignoring the fact that there are many other server that are also connected to it. Such mechanism of allocation of server eventually disrupts scheduling performance.

- Least Significance towards HARQ: There are various literatures published in past decade discussing about the benefits of using Hybrid Automatic Repeat Request for facilitating the reliability of transmission in wireless networks. It does so by harnessing the errors occurring during transmission. The mechanism of HARQ scheme by using feedbacks actually assists the node to take much better decision of scheduling. However, irrespective of its beneficial features, HARQ is not found to be investigating in connection with the scheduling practices in existing research community towards LTEbased networks.

- Rare Benchmarked Model: The existing research-based approaches towards improving communication performance in LTE are highly scattered and less work towards scheduling has been carried out. However, the existing amount of studies towards scheduling are not reported to be benchmarked or standardized that reduces the possibility of practical utilization until further research is continued. Inclusion of exhaustive traffic model, test scenarios in LTE are less considered in existing scheduling approaches.

The above stated research gap are found and considered to be addressed in existing system.

\section{CONCLUSION \& FUTURE WORK}

The prime intention of the proposed study was to study the existing research work being carried out towards leveraging the scheduling process in LTE-based networks. It has been noticed that LTE uses two different access schemes explicitly for uplink (OFDM/OFDMA) as well as for downlink (SC-FDMA) transmission. The paper has discussed LTE significances theoretically followed by existing research-based solutions. Although, there has been various studies carried out towards scheduling techniques in LTE, still there are open-end problems. Almost all the existing approaches are associated with limitations. It was also observed that there is rising level of interest towards improving the performance of throughput and delay; however, existing studies are not found to offer any such robust solution for this. While there existing various techniques but very few of them are found to address the practicality of implementation, which is the major flaw and set back of majority of existing approaches. Hence, this paper contributes to i) offer a quick snapshot of LTE-based network, ii) compact discussion of some recent implementation work towards scheduling, iii) identifying the advantage and limitations associated with each implementation, and iv) discussion the open -end problems in terms of research gap.

The future work will be definitely towards bridging the research gap being identified in this paper. The first approach will be to develop a model that offers scheduling for improving both throughput and

APTIKOM J. CSIT Vol. 3, No. 2, 2018: $51-58$ 
delay performance at same time considering all sorts of practical constraints in LTE-based network. The second possible future work could be to include the hybrid ARQ mechanism to boost up the channel efficiency of LTE, which will indirectly improve the communication performance too.

\section{REFERENCES}

[1] B Furht, Syed A Ahson. Long Term Evolution: 3GPP LTE Radio and Cellular Technology. 2016.

[2] H Zhang, Sami Khairy, Lin X Cai, Zhu Han. Resource Allocation in Unlicensed Long Term Evolution HetNets, Springer. 2017.

[3] S Sesia, Matthew Baker, Issam Toufik. LTE - The UMTS Long Term Evolution: From Theory to Practice. John Wiley \& Sons. 2011.

[4] A Paradisi, Michel Daoud Yacoub, Fabrício Lira Figueiredo, Tania Tronco. Long Term Evolution: 4G and Beyond, Springer. 2015.

[5] K Pahlavan, Prashant Krishnamurthy, Principles of Wireless Access and Localization. John Wiley \& Sons. 2013.

[6] A Yahya. LTE-A Cellular Networks: Multi-hop Relay for Coverage, Capacity and Performance Enhancement, Springer. 2016.

[7] Q-D Ho, Daniel Tweed, Tho Le-Ngoc. Long Term Evolution in Unlicensed Bands. Springer. 2016.

[8] Panagopoulos, Athanasios D. Handbook of Research on Next Generation Mobile Communication Systems. IGI Global. 2015.

[9] M Rupp, Stefan Schwarz, Martin Taranetz. The Vienna LTE-Advanced Simulators: Up and Downlink, Link and System Level Simulation. Springer. 2016.

[10] M Danilo Abrignani, L. Giupponi, A. Lodi, R Verdone. Scheduling M2M traffic over LTE uplink of a dense small cells network. 2015 International Symposium on Wireless Communication Systems (ISWCS). Brussels. 2015: 760-764.

[11] RR Su, IS Hwang. Efficient resource allocation scheme with grey relational analysis for the uplink scheduling of 3GPP LTE networks. 2016 IEEE International Conference on Industrial Technology (ICIT). 2016: 599-603.

[12] J Park, Jaemin Ahn. Improving frequency multiplexing of random access resources for TDD system enhancement. 2016 Eighth International Conference on Ubiquitous and Future Networks (ICUFN). 2016: 661-666.

[13] M Ayhan, L Zappaterra, HA Choi. Geometric average-based fast-converging proportional fair scheduling for LTE downlink transmissions. 2016 1st International Workshop on Link- and System Level Simulations (IWSLS). 2016: 1-6.

[14] VC Gungor, OG Uyan. QoS-aware downlink scheduling algorithm for LTE networks: A case study on edge users. 2017 25th Signal Processing and Communications Applications Conference (SIU). 2017: 1-4.

[15] H Shan, Y Zhang, W Zhuang, A Huang, Z Zhang. User Behavior-Aware Scheduling Based on Time-Frequency Resource Conversion. in IEEE Transactions on Vehicular Technology. 2017; 66(9): 8429-8444.

[16] HAM Ramli, MK Hasan, AF Ismail, AH Abdalla, K Abdullah. An investigation of packet scheduling algorithms for Long Term Evolution-Advanced. 2012 IEEE Conference on Open Systems. 2012: 1-5.

[17] HW Tseng, WC Lee, CR Sheu, YH Lee, YG Jan. Applying genetic algorithms to the data traffic scheduling and performance analysis of a long-term evolution system. 2012 International Symposium on Intelligent Signal Processing and Communications Systems. 2012: 183-188.

[18] O Ergul, O Yilmaz, AT Koc, OB Akan. DRX and QoS-aware energy-efficient uplink scheduling for long term evolution. 2013 IEEE Global Communications Conference (GLOBECOM). 2013: 4644-4649.

[19] AD Maleki, B Abolhassani. New scheduling scheme for green communications in long term evolution networks. in IET Communications. 2014; 8(14): 2438-2444.

[20] JG Neto, FACM Cardoso, PAV Ferreira, MHM Costa. Customizable algorithm for data scheduling in long term evolution networks. 2015 Information Theory and Applications Workshop (ITA). 2015: 123-129.

[21] S-B Lee, S Choudhury, A Khoshnevis, S Xu, S Lu. Downlink MIMO with frequency-domain packet scheduling for 3GPP LTE. in Proceedings of the 28th IEEE Conference on Computer Communications. 2009: 2611-2615.

[22] N Wei, A Pokhariyal, TB Sørensen, TE Kolding, PE Mogensen. Performance of MIMO with frequency domain packet scheduling in UTRAN LTE downlink. in Proceedings of the IEEE Vehicular Technology Conference (VTC'07). 2007: 1177-1181.

[23] G Aiyetoro, F Takawira, T Walingo. Near-optimal packet scheduling scheme in satellite LTE networks. in IET Communications. 2017; 11(15): 2311-2319.

[24] Y Chen, G Liu. Playout buffer and DRX aware scheduling scheme for video streaming over LTE system. in IET Communications. 2016; 10(15): 1971-1978.

[25] J Fan, D Lee, GY Li, L Li. Multiuser Scheduling and Pairing With Interference Mitigation for LTE Uplink Cellular Networks. in IEEE Transactions on Vehicular Technology. 2015; 64(2): 481-492.

[26] N Ferdosian, M Othman, BM Ali, K Yeah Lun. Multi-Targeted Downlink Scheduling for Overload-States in LTE Networks: Proportional Fractional Knapsack Algorithm with Gaussian Weights. in IEEE Access. 2017; 5: 30163027.

[27] F Ghavimi, YW Lu, HH Chen. Uplink Scheduling and Power Allocation for M2M Communications in SCFDMA-Based LTE-A Networks with QoS Guarantees. in IEEE Transactions on Vehicular Technology. 2017; 66(7): 6160-6170. 
[28] O Grøndalen, A Zanella, K Mahmood, M Carpin, J Rasool, ON Østerbø. Scheduling Policies in Time and Frequency Domains for LTE Downlink Channel: A Performance Comparison. in IEEE Transactions on Vehicular Technology. 2017; 66(4): 3345-3360.

[29] W Guo, J Fan, GY Li, Q Yin, X Zhu. Adaptive SU/MU-MIMO scheduling schemes for LTE-A downlink transmission. in IET Communications. 2017; 11(6): 783-792.

[30] H Ko, J Lee, S Pack. Joint Optimization of Channel Selection and Frame Scheduling for Coexistence of LTE and WLAN. in IEEE Transactions on Vehicular Technology. 2018; 67(7): 6481-6491.

[31] GY Li, J Niu, D Lee, J Fan, Y Fu. Multi-Cell Coordinated Scheduling and MIMO in LTE. in IEEE Communications Surveys \& Tutorials. 2014; 16(2): 761-775.

[32] SY Lien, J Lee, YC Liang. Random Access or Scheduling: Optimum LTE Licensed-Assisted Access to Unlicensed Spectrum. in IEEE Communications Letters. 2016; 20(3): 590-593.

[33] Q Liu, CW Chen. Smart Downlink Scheduling for Multimedia Streaming Over LTE Networks with Hard Handoff. in IEEE Transactions on Circuits and Systems for Video Technology. 2015; 25(11): 1815-1829.

[34] Z Luo, M Ding, H Luo. CC On/Off Scheduling Using Learning-Based Prediction for LTE in the Unlicensed Spectrum. in IEEE Communications Letters. 2015; 19(12): 2158-2161.

[35] MA Mehaseb, Y Gadallah, A Elhamy, H Elhennawy. Classification of LTE Uplink Scheduling Techniques: An M2M Perspective. in IEEE Communications Surveys \& Tutorials. 2016; 18(2): 1310-1335.

[36] M Minelli, M Ma, M Coupechoux, P Godlewski. Scheduling Impact on the Performance of Relay-Enhanced LTEA Networks. in IEEE Transactions on Vehicular Technology. 2016; 65(4): 2496-2508.

[37] AE Mostafa, Y Gadallah. A Statistical Priority-Based Scheduling Metric for M2M Communications in LTE Networks. in IEEE Access. 2017; 5: 8106-8117.

[38] MS Pan, TM Lin, CY Chiu, CY Wang. Downlink Traffic Scheduling for LTE-A Small Cell Networks with Dual Connectivity Enhancement. in IEEE Communications Letters. 2016; 20(4): 796-799.

[39] A Ragaleux, S Baey, M Karaca. Standard-Compliant LTE-A Uplink Scheduling Scheme with Quality of Service. in IEEE Transactions on Vehicular Technology. 2017; 66(8): 7207-7222.

[40] YC Wang, TY Tsai. A Pricing-Aware Resource Scheduling Framework for LTE Networks. in IEEE/ACM Transactions on Networking. 2017; 25(3): 1445-1458.

[41] A Zolfaghari, H Taheri. Queue-Aware Channel-Adapted Scheduling and Congestion Control for Best-Effort Services in LTE Networks. in Canadian Journal of Electrical and Computer Engineering. 2015; 38(2): 170-182.

[42] A Zolfaghari, H Taheri. Joint Best Price-CQI Product Scheduling and Congestion Control for LTE. in Canadian Journal of Electrical and Computer Engineering. 2016; 39(4): 255-267.

[43] R Molina-Masegosa, J Gozalvez. System Level Evaluation of LTE-V2V Mode 4 Communications and Its Distributed Scheduling. 2017 IEEE 85th Vehicular Technology Conference (VTC Spring). 2017: 1-5.

[44] BJ Chang, PY Chang, YH Liang. Adaptive packet scheduling and radio RB allocation for LTE-A toward 5G relaying communications. 2017 8th IEEE Annual Information Technology, Electronics and Mobile Communication Conference (IEMCON). Vancouver, BC. 2017: 358-364.

[45] Y Kim, S Park. Analytical Calculation of Spectrum Requirements for LTE-A Using the Probability Distribution on the Scheduled Resource Blocks. in IEEE Communications Letters. 2018; 22(3): 602 - 605.

[46] A Chakrapani. Efficient resource scheduling for eMTC/NB-IoT communications in LTE Rel. 13. 2017 IEEE Conference on Standards for Communications and Networking (CSCN). 2017: 66-71.

[47] F Feng, F Peng, B Yan, S Lin, J. Zhang. QoS-based LTE downlink scheduling algorithm for smart grid communication. 2017 IEEE 9th International Conference on Communication Software and Networks (ICCSN). 2017: 548-552.

[48] Y Alaa, HM ElAttar, F Digham, LH Afify, H Elbadawy. LTE dynamic scheduling scheme for massive M2M and H2H communication. 2017 IEEE 8th Annual Ubiquitous Computing, Electronics and Mobile Communication Conference (UEMCON). 2017: 478-482.

[49] CP Lau, A Alabbasi, B Shihada. An Efficient Live TV Scheduling System for 4G LTE Broadcast. in IEEE Systems Journal. 2017; 11(4): 2737-2748.

[50] C Cano, DJ Leith, A Garcia-Saavedra, P Serrano. Fair Coexistence of Scheduled and Random Access Wireless Networks: Unlicensed LTE/WiFi. in IEEE/ACM Transactions on Networking. 2017; 25(6): 3267-3281.

[51] AS Eladham, N ElShennawy, A Sarhan. Modified capacity enhanced PF (MCE-PF) downlink scheduling algorithm for heterogeneous LTE-Advanced Networks. 2017 13th International Computer Engineering Conference (ICENCO). 2017: 1-7.

[52] RO Xitlaly, GRJ María, VC Norman. Analysis of the throughput performance for a LTE-A network in Managua using the strategies of the Packet Scheduling and frequency bands 1, 2 and 3 FDD," 2017 IEEE 37th Central America and Panama Convention (CONCAPAN XXXVII). 2017: 1-6.

APTIKOM J. CSIT Vol. 3, No. 2, 2018: 51-58 